\title{
Tracing Tubular Objects in 3D Confocal Images Using Haptic Device.
}

\author{
Jiří Janáček $^{1}$, Lucie Kubínová ${ }^{1}$ and Xiao Wen Mao $^{2}$ \\ 1. Department of Biomathematics, Institute of Physiology ASCR, Prague, Czech Republic \\ 2. Department of Radiation Medicine, Loma Linda University, Loma Linda, CA, USA
}

Capillary net consists of tubules connected in complicated network. It can be modeled by network of lines in $3 \mathrm{D}$ and described by quantitative characteristics such as length, orientation and connectivity. The characteristics influence the physiological properties of the capillary bed and provide necessary parameters for physical models of the transport processes in tissues [3].

Blood capillaries in brain [5] and skeletal muscle [1] can be visualized and studied in 3D by confocal microscopy. 3D data enable direct analysis of the geometric properties of complex networks by image analysis techniques. The graphs of capillaries that cannot be detected automatically due to low contrast, high noise or complicated background but are clearly visible can still be digitized manually using a 3D editing program. We use a custom made program for PC with $3 \mathrm{D}$ interface. The interface consists of stereoscopic volume rendering of images with 3D cursor with 6 degrees of freedom (DOF) controlled by a haptic device (SensableOmni, SenseGraphics AB, Sweden) with 3 DOF force proportional to the gradient of the image intensity. The haptic feedback serves as a cue for depth perception and allows better placement of cursor in the capillaries. The program enables 3D drawing of capillaries as chains of line segments, moving individual points, connecting the chains together or splitting them and deleting the vertices or whole chains. All operations are stored in a queue and can be reversed by undo/redo commands. The virtual camera position is controlled interactively by mouse or by 6 DOF device (Space Navigator, 3dconnexion, USA).

The stereoscopic rendering is accomplished either by a special graphic card with a dedicated monitor and LCD shutter glasses or by anaglyph visualization using a common display and color (RB) glasses. As the rendering of the cursor with the whole scene containing volume data would be too slow, the cursor is rendered separately and drawn on top of the rendered views of the scene in XOR mode. Thus the time hierarchy of the program response is $1 \mathrm{~ms}$ for haptic feedback, about $20 \mathrm{~ms}$ for cursor rendering and haptic force setting and typically $0.1 \mathrm{~s}$ for volume rendering. The program was written in $\mathrm{C}++$ in Visual studio 2008 (Microsoft).

Alternatively other 3D editors such as Filament editor in the Amira program (FEI) can be used for capillaries delineation. The representation of the lineal structure by 3D graph is suitable for direct calculation of the length as well as of topological and orientation characteristics. The length of capillaries is the sum of the lengths of line segments. The course of capillaries can be described by connectivity and the degree of anisotropy (capillaries orientation).

The connectivity of the capillary bed can be characterized by Euler-Poincaré characteristic $\chi$ [2]. It can be calculated as $\sum_{i}\left(2-d_{i}\right) / 2$ where $d_{i}$ is degree of $\mathrm{i}$-th branching point or endpoint. In typical samples of capillary network, i.e. for connected network without endpoints and with triple branching points only the number of capillary branching is an estimate of the value $-2 \chi$. 
Orientation of capillaries can be quantified by the degree of anisotropy computed from eigenvalues $\lambda_{3} \geq \lambda_{2}$ $\geq \lambda_{1} \geq 0$ of mean structural tensor [4] calculated as length weighted average $T=\sum_{i} L_{i} U_{i} / \sum_{i} L_{i}$ where the structural tensor of individual segment $U=v v^{T}$ is calculated from its direction $v=(b-a) / L$ where $a$ and $b$ are endpoints of the segment and $L$ is its length.

Stack of images of optical sections of choroid tissue stained by intravenous injection of biotin-labeled Lycopersicon esculentum lectin were captured by Olympus Fluoview confocal microscope with planapochromatic $60 x$ oil objective with NA 1.42 at excitation wavelength $488 \mathrm{~nm}$ with axial step $1 \mu \mathrm{m}$.

Total length of the capillaries is $2.43 \mathrm{~mm}$, Euler characteristics of the capillary bed is -51 , there are 98 branchings ( 93 triple, 5 quadruple) and 1 endpoint. The values of eigenvalues estimates were $\lambda_{1}=0.593, \lambda_{2}$ $=0.521, \lambda_{3}=0.296$. Obviously the capillary bed is flat (because $\lambda_{2}>>\lambda_{1}$ ) with no observable anizotropy in the plane (because $\lambda_{3} \cong \lambda_{2}$ ).

The low-cost haptic device enables precise manipulation with 3D data volume; however it was not implemented in available microscopy imaging software yet. In addition to editing 3D data structures shown in this paper, the device can be used for real time tasks as the control of optical tweezer for nanoparticles manipulation.

\section{References:}

[1] V Čebašek et al, Microvasc Res 79 (2010), p. 40-46.

[2] HJG Gundersen et al, Bone 14 (1993), p. 217-222.

[3] JW Ji et al, J Theor Biol 241 (2006), p. 94-108.

[4] P Kochová et al, J Theor Biol 286 (2011), p. 67-78.

[5] L Kubínová, XW Mao, J Janáček, Microsc Microanal 19 (2013), p. 898-906.

[6] The authors acknowledge funding from the Czech Republic's public funds provided by Academy of Sciences (AV0Z50110509 and RVO:67985823), Ministry of Education, Youth and Sports (KONTAKT LH13028), and Science foundation of the Czech Republic (13-12412S).
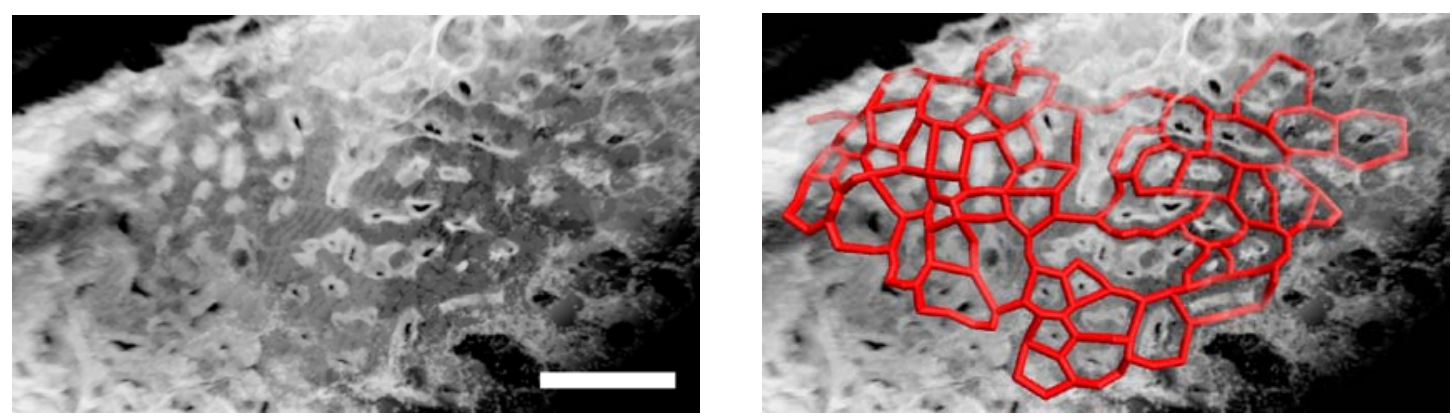

Figure 1. Volume rendering of confocal stack (right). The same view with tubular model of choroid capillaries axes (left). The scalebar is $50 \mu \mathrm{m}$ long. 